\title{
Prognostic Significance of EMVI in Rectal Cancer in a Tertiary Cancer Hospital in India
}

\author{
Sayali Y. Pangarkar ${ }^{1,2}$ Akshay D. Baheti ${ }^{1}$ Kunal A. Mistry ${ }^{1,2}$ Amit J. Choudhari ${ }^{1,2}$ \\ Vasundhara R. Patil ${ }^{1,2}$ Ankita Ahuja ${ }^{1,2}$ Aparna N. Katdare ${ }^{1,2} \quad$ Akshay B. Patil $^{3}$ Vikas S. Ostwal ${ }^{2,4}$ \\ Mukta R. Ramadwar ${ }^{2,5}$ Reena Z. Engineer ${ }^{2,6}$ Avanish P. Saklani ${ }^{2,7}$
}

${ }^{1}$ Department of Radio-diagnosis, Tata Memorial Centre, Mumbai, Maharashtra, India

2 Homi Bhabha National Institute, Mumbai, Maharashtra, India

${ }^{3}$ Division of Clinical Research and Statistics, Tata Memorial Hospital,

Mumbai, Maharashtra, India

${ }^{4}$ Department of Medical Oncology, Tata Memorial Centre, Mumbai,

Maharashtra, India

${ }^{5}$ Department of Pathology Tata Memorial Centre, Mumbai,

Maharashtra, India

Indian J Radiol Imaging 2021;31:560-565.
Address for correspondence Akshay D. Baheti, MD, Department of Radio-diagnosis, Tata Memorial Hospital, Dr Ernest Borges Rd, Parel East, Parel, Mumbai, Maharashtra 400012, India

(e-mail: akshaybaheti@gmail.com).

${ }^{6}$ Department of Radiation Oncology, Tata Memorial Centre, Mumbai, Maharashtra, India

${ }^{7}$ Department of Surgical Oncology, Tata Memorial Centre, Mumbai, Maharashtra, India

\begin{abstract}
Background Presence of extramural venous invasion (EMVI) is a poor prognostic factor for rectal cancer as per literature. However, India-specific data are lacking.

Aim The aim of the study is to determine the prognostic significance of EMVI in locally advanced rectal cancer on baseline MRI.

Materials and Methods We retrospectively reviewed 117 MRIs of operable nonmetastatic locally advanced rectal cancers in a tertiary cancer institute. Three dedicated oncoradiologists determined presence or absence of EMVI, and its length and thickness, in consensus. These patients were treated as per standard institutional protocols and followed up for a median period of 37 months (range: 2-71 months). Kaplan-Meier curves $(95 \% \mathrm{Cl})$ were used to determine disease-free survival (DFS), distant-metastases free survival (DMFS), and overall survival (OS). Univariate analysis was performed by comparing groups with log-rank test.

Results EMVI positive cases were 34/114 (29\%). More EMVI-positive cases developed distant metastasis compared with EMVI-negative cases (14/34-41\% vs. 22/83-26\%). The difference, however, was not statistically significant $(p=0.146)$. After excluding signet-ring cell cancers $(n=14)$, EMVI showed significant correlation with DMFS $(p=0.046)$, but not with DFS or OS. The median thickness and length of EMVI was 6 and $14 \mathrm{~mm}$, respectively in

Keywords

- extramural venous invasion

- MRI

- rectal cancer patients who developed distant metastasis, as compared with 5 and $11 \mathrm{~mm}$ in those who did not, although this difference was not statistically significant.

Conclusion EMVI is a predictor of distant metastasis in locally advanced nonmetastatic, non-signet ring cell rectal cancers. EMVI can be considered another high-risk feature to predict distant metastasis.
\end{abstract}

DOI https://doi.org/ $10.1055 / \mathrm{s}-0041-1736404$ ISSN 0971-3026.
(C) 2021. Indian Radiological Association. All rights reserved. This is an open access article published by Thieme under the terms of the Creative Commons Attribution-NonDerivative-NonCommercial-License, permitting copying and reproduction so long as the original work is given appropriate credit. Contents may not be used for commercial purposes, or adapted, remixed, transformed or built upon. (https://creativecommons.org/ licenses/by-nc-nd/4.0/)

Thieme Medical and Scientific Publishers Pvt. Ltd., A-12, 2nd Floor, Sector 2, Noida-201301 UP, India 


\section{Introduction}

Colorectal cancer is a common cancer affecting the Indian population, with up to $30 \%$ being upfront metastatic. ${ }^{1,2}$ The signet ring cell variety of rectal carcinoma is particularly aggressive, with a high propensity for peritoneal metastasis and involvement of a younger age group.,4 Although the worldwide incidence of this histologic variant is $1 \%$, Indian population shows a higher incidence varying from 11 to $13 \%$ in different studies. ${ }^{2,5}$

Extramural venous invasion (EMVI) i.e., spread of tumor into perirectal vessels, is an established risk factor for distant metastasis, with reduced disease-free survival (DFS) and overall survival (OS) ${ }^{6-8}$ Our literature search did not show any imaging study in the Indian population with regards to the significance of EMVI in rectal cancer. This would be interesting to see because of the frequently seen advanced disease at presentation and the higher proportion of signet ring cell cases in the Indian population, increasing the confounding factors for poor outcomes. Furthermore, while EMVI has prognostic significance, it is possible that patients with thicker and longer extent of involvement have a worse prognosis compared with patients with mild involvement. ${ }^{8,9}$ Thus, the objective of our study was to determine the prognostic significance of EMVI in rectal cancers in the Indian population, and to evaluate whether the length and thickness of involvement correlated with prognosis.

\section{Materials and Methods}

\section{Subjects}

This is an Institutional Review Board approved retrospective study. We identified 166 consecutive cases of operable nonmetastatic pathologically proven rectal cancer from our colorectal disease management group database from the year 2011 to 2015. Patients presenting with non-metastatic rectal cancer and baseline MRI available were included in the study. MRI performed in outside institutes was included if DICOM images were available on PACS. Exclusion criteria were suboptimal image quality, patients that progressed during the neoadjuvant chemoradiation and did not undergo curative treatment, and patients lost to follow-up.

\section{Clinical and Histopathology Data}

The patient demographic data, histopathology, treatment, and follow-up details were extracted from the institutional electronic medical records. All definitive surgeries were performed by dedicated gastrointestinal oncosurgeons at our institute. All patients were treated with neoadjuvant chemoradiation (CTRT) followed by definitive surgery and were followed up for a median period of 37 months (range: 2-71 months).

\section{Imaging and Image Analysis}

MR images were acquired on 1.5T (GE Healthcare, Milwaukee, United States), 1.5T (Philips Medical Systems, Eindhoven, Netherlands), or 3T (GE Healthcare, Milwaukee, United States) machines in our institute using the institu- tional rectal MRI protocol. This included large FOV (field of view) T1W and T2W axial, T2W sagittal sequences of the pelvis, small FOV thin section oblique axial T2W sequence perpendicular to the plane of the rectal tumor and oblique coronal $\mathrm{T} 2 \mathrm{~W}$ sequence parallel to the plane of the tumor. No intravenous contrast was administered as per institutional protocol. For contrast-enhanced MRI studies performed outside, the contrast-enhanced sequences were not assessed.

The images were retrospectively and systematically reviewed on Centricity PACS (GE Healthcare, Milwaukee, United States) workstation by three oncoradiologists in consensus, with 11,9 , and 4 years of experience, respectively, who were blinded to the histopathology findings and patient outcome. EMVI was identified by expansion or irregularity of vessels, loss of normal vascular flow void and intraluminal intermediate tumor signal intensity, contiguous or separate from the main tumor. ${ }^{10}$ EMVI was graded with a 0 to 4 scoring system as suggested by Jhaveri et al. ${ }^{11}$ Score 0 denoted no vessel in vicinity of extramural tumor penetration, score 1 denoted vessels with normal caliber and without definite tumor signal intensity, score 2 denoted slightly expanded vessels without definite tumor signal intensity, score 3 denotes intermediate tumor signal intensity within expanded vessels, and score 4 denotes obvious irregular vessel contour or nodular expansion of vessel by definite tumor signal (-Figs. 1 and 2). For the purpose of calculation, scores 0 to 2 were considered negative for EMVI, while scores 3 and 4 were considered positive. The length and the thickness of the involved vessel were also recorded (-Fig. 3). Tumor within $1 \mathrm{~mm}$ of the mesorectal fascia was considered CRM (circumferential resection margin) positive.

\section{Statistical Analysis}

Statistical analysis was performed using SPSS (the statistical package for social sciences), IBM Corp, released 2017, IBM SPSS Statistics for Windows, Version 25.0. Armonk, NY: IBM Corp. Kaplan-Meier curves $(95 \% \mathrm{CI})$ were used to determine DFS, distant-metastases free survival (DMFS), and OS. Univariate analysis was performed by comparing groups with log-rank test. All analysis was two sided and significance was set at $p$-value of 0.05 .

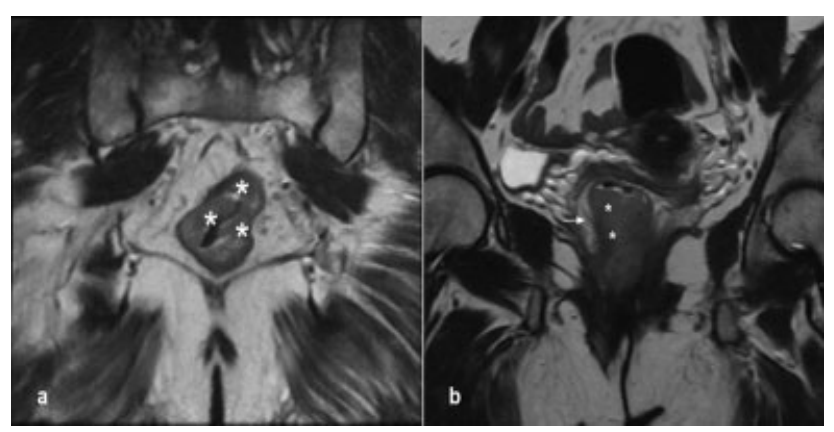

Fig. 1 (a) Coronal T2-weighted image showing rectal wall thickening $\left({ }^{*}\right)$ with no vessel in the vicinity of the tumor, Grade 0 EMVI. (b) Coronal T2-weighted image showing rectal tumor $\left({ }^{*}\right)$ with a normal caliber vessel in its vicinity (white arrow), Grade $1 \mathrm{EMVI}$. EMVI, extramural venous invasion. 


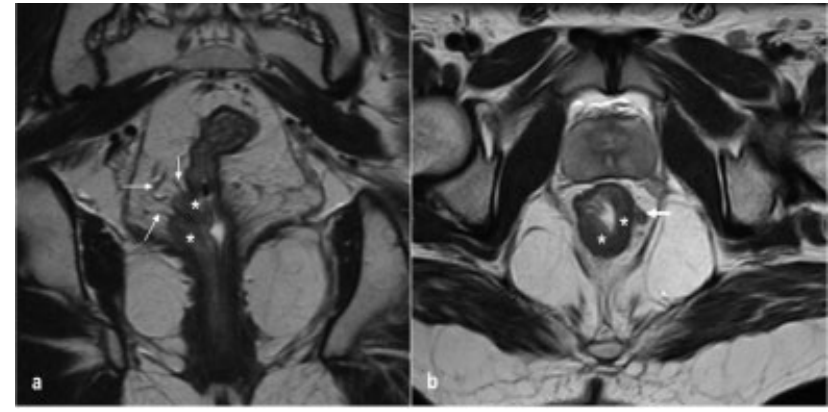

Fig. 2 (a) Coronal T2-weighted image showing rectal tumor $\left({ }^{*}\right)$ with a prominent normal-appearing vessel in its vicinity, Grade 2 EMVI. (b) Axial T2-weighted image showing rectal tumor $\left({ }^{*}\right)$ with an expanded vessel showing regular contour and tumor signal intensity within its lumen (white arrow), Grade 3 EMVI. EMVI, extramural venous invasion.

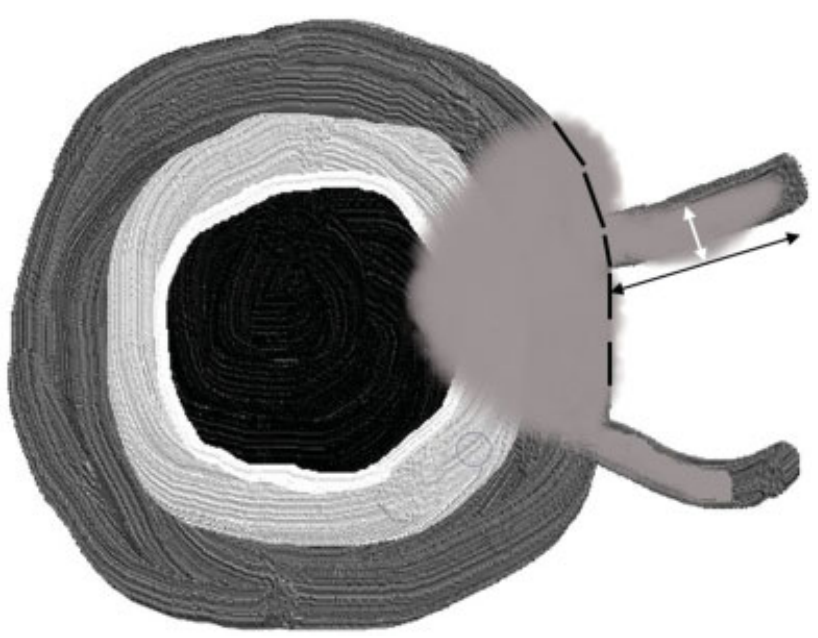

Fig. 3 Illustration showing measurement of maximum length (black double arrow) and thickness (white double arrow) of EMVI. EMVI, extramural venous invasion.

\section{Results}

A total of 117 patients were included in the study (-Fig. 4). $45 / 117$ (38.5\%) cases were females and 72 (61.5\%) were males with a median age of 51 years. The baseline characteristics of the patients are given in - Table 1. EMVI was present in 34/117 (29\%) cases and absent in $83(71 \%)$ cases. Twelve patients had grade 3 EMVI and 22 had grade 4 EMVI. Overall,

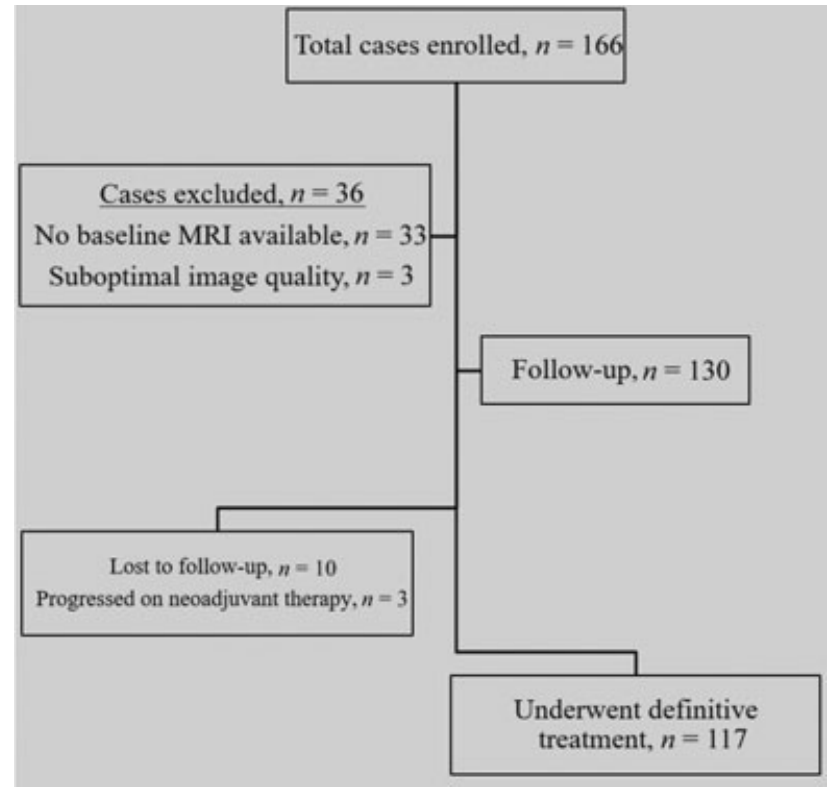

Fig. 4 Consort diagram of case selection.

Table 1 Baseline characteristics of primary tumor determined on MRI

\begin{tabular}{|l|l|l|}
\hline $\begin{array}{l}\text { Baseline } \\
\text { characteristics }\end{array}$ & Stage & $\begin{array}{l}\text { No. of patients } \\
\text { out of } \boldsymbol{n}=\mathbf{1 1 7}\end{array}$ \\
\hline mrT stage & $<\mathrm{T3}$ & $6(5 \%)$ \\
\hline & T3 & $91(78 \%)$ \\
\hline & T4 & $20(17 \%)$ \\
\hline mrN stage & N0 & $25(21 \%)$ \\
\hline & N1 & $51(44 \%)$ \\
\hline & N2 & $41(35 \%)$ \\
\hline mrCRM status & Positive & $63(53.9 \%)$ \\
\hline & Negative & $54(46.1 \%)$ \\
\hline
\end{tabular}

36/117 (30\%) developed distant metastasis. 15/117 (12.8\%) developed local recurrence, with $8 / 15$ of these also having distant metastasis (- Table 2). Three-year DFS and OS were 67 and $82 \%$, respectively in EMVI positive cases, compared with 74 and $82 \%$ in EMVI negative cases. However, this did not reach statistical significance (-Table 3; - Figs. 5 and $\mathbf{6}$ ).

Table 2 Distribution of EMVI positive and negative cases and their correlation to distant metastases, local recurrence, and survival

\begin{tabular}{|l|l|l|l|l|l|l|l|}
\hline EMVI status & $\begin{array}{l}\text { No. of } \\
\text { cases }\end{array}$ & $\begin{array}{l}\text { Grade of } \\
\text { EMVI }\end{array}$ & $\begin{array}{l}\text { No. of } \\
\text { cases }\end{array}$ & $\begin{array}{l}\text { Distant metastasis } \\
\text { Grade-wise }\end{array}$ & $\begin{array}{l}\text { Distant metastases } \\
\text { (DM) Total }\end{array}$ & $\begin{array}{l}\text { Local recurrence } \\
\text { (LR) Total }\end{array}$ & $\begin{array}{l}\text { Deaths total } \\
\text { Negative }\end{array}$ \\
\hline $83 / 117$ & 0 & 24 & 7 & $22 / 83(26 \%)$ & $10 / 83(12 \%)$ & $11 / 83(13 \%)$ \\
\hline & & 1 & 49 & 10 & & & \\
\hline & & 2 & 10 & 5 & & & \\
\hline Positive & $34 / 117$ & 3 & 12 & 2 & $14 / 34(41 \%)$ & $5 / 34(14.7 \%)$ & $7 / 34(20.5 \%)$ \\
\hline & & 4 & 22 & 12 & & & \\
\hline
\end{tabular}


Table 3 Three-year DFS and OS in EMVI positive and negative cases in entire study population and in the signet ring cell negative subset

\begin{tabular}{|l|l|l|l|l|l|}
\hline Patients & EMVI & $3-y$ DFS & $p$ & $3-y$ OS & $p$ \\
\hline All cases $(n=117)$ & Negative & $74.2 \%$ & 0.180 & $87 \%$ & 0.211 \\
\hline & Positive & $67.2 \%$ & & $82 \%$ & \\
\hline Signet ring cell negative cases $(n=103)$ & Negative & $79 \%$ & 0.109 & $91 \%$ & 0.22 \\
\cline { 2 - 6 } & Positive & $72 \%$ & & $88.6 \%$ \\
\hline
\end{tabular}

Abbreviations: DFS, disease-free survival; EMVI, extramural venous invasion; OS, overall survival.

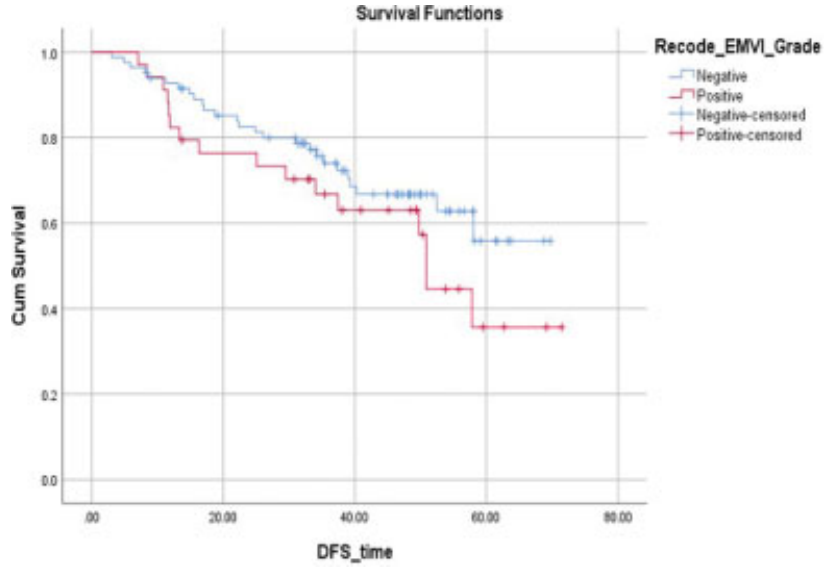

Fig. 5 Plot of Kaplan-Meier curve $(95 \% \mathrm{Cl})$ estimating disease free survival (DFS) in EMVI negative and positive cases in all patients $(n=117) ; p=0.211$. EMVI, extramural venous invasion.

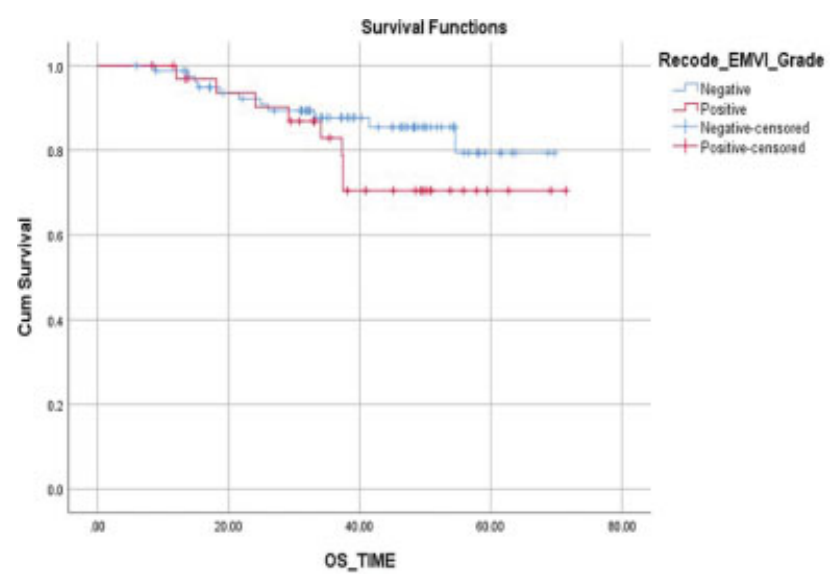

Fig. 6 Plot of Kaplan-Meier curve $(95 \% \mathrm{Cl})$ of overall survival $(\mathrm{OS})$ in EMVI negative and positive cases in all patients $(n=117) ; p=0.211$. EMVI, extramural venous invasion.

Fourteen out of 34 (41\%) EMVI-positive cases developed distant metastasis, compared with 22/83 (26\%) EMVI-negative cases (-Table 2 ). The difference, however, was not statistically significant $(p=0.146)$. After excluding signetring cell positive cases $(n=14)$, presence of EMVI showed significant correlation with distant metastasis free survival $(p=0.046)$ (-Fig. 7), with $12 / 29(41 \%)$ cases with EMVI developing metastases compared with 16/74 (22\%) patients

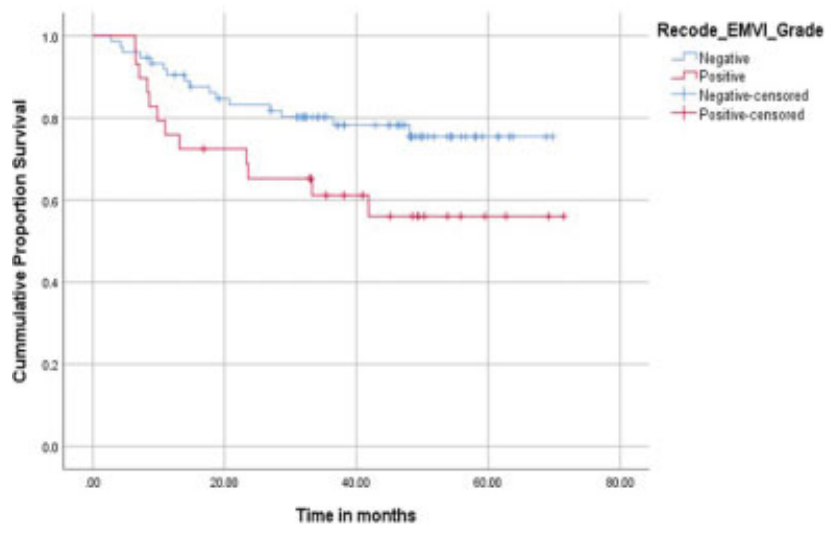

Fig. 7 Plot of Kaplan-Meier curve $(95 \% \mathrm{Cl})$ of distance metastasis free survival (DMFS) in EMVI negative and positive cases (in signet-ring cell negative cases, $n=103$ ); $p=0.046$. EMVI, extramural venous invasion.

without EMVI. However, there was no statistical difference in the overall DFS and OS (- Table 3 ).

The median thickness of EMVI was $6 \mathrm{~mm}$ and median length of EMVI was $14 \mathrm{~mm}$ in patients who developed distant metastasis, compared with 5 and $11 \mathrm{~mm}$, respectively, in patients who did not develop distant metastasis. This was not statistically significant.

\section{Discussion}

MRI is the imaging modality of choice for staging and restaging of rectal cancer. The prognosis of rectal cancer in terms of survival and likelihood of recurrence depends on several factors such as histologic grade, $\mathrm{T}$ category, $\mathrm{N}$ category, CRM involvement, and EMVI. ${ }^{12-15}$

Traditionally, venous invasion was a histopathological concept. ${ }^{16}$ Many studies have shown pathological venous invasion to portend worse prognosis. ${ }^{17,18}$ A systematic review by Chand et al $^{19}$ of 14 pathological studies demonstrates venous invasion and more specifically EMVI to be associated with worse survival. The 5-year survival ranged from 20 to 33\% in EMVI positive cases to 45 to $73 \%$ in EMVI negative cases. ${ }^{16,20,21}$

MRI has moderate sensitivity and good specificity for detecting EMVI. ${ }^{22}$ Multiple studies have demonstrated that mrEMVI correlates with worse prognosis. ${ }^{6,8,23-26}$ A recent meta-analysis of 1,262 patients demonstrated a prevalence of mrEMVI in about one-third patients, similar to our study. ${ }^{6}$ 
They found an almost fourfold increase in the risk of developing metastases in patients with mrEMVI present at baseline. Various studies demonstrate development of distant metastases in 6.7 to $23 \%$ mrEMVI negative cases compared with 24.5 to $55 \%$ in mrEMVI positive cases. ${ }^{8,24,27}$ Our Indiaspecific data shows a similar trend, with $26 \%$ mrEMVI negative and $41 \%$ mrEMVI positive developing distant metastases. The percentage of mrEMVI negative patients developing subsequent metastatic recurrence is slightly higher than other studies; the reasons for this could include the higher incidence of signet cell tumors in the Indian population and possible relatively advanced tumors at the time of presentation.

Gu et $\mathrm{al}^{28}$ found a 3 -year DFS of $60 \%$ for mrEMVI scores 3 and 4 and $86 \%$ with mrEMVI scores 0 to 2 . Similarly, in a study of 142 patients, Smith et al $^{25}$ observed a DFS of $35 \%$ at 3 years for patients with mrEMVI scores of 3 to 4 , compared with $74 \%$ for those with mrEMVI 0 to 2. Our study results showed a 3year DFS of $67 \%$ for EMVI 3 to 4 and $74 \%$ for EMVI grades 0 to 2 , although this was not statistically significant.

There is mixed data with regards to correlation of mrEMVI with OS, with some studies not finding statistically significant correlation. ${ }^{28,29}$ While others demonstrating significantly worsened survival in mrEMVI positive patients, ${ }^{24,27}$ our study findings showed a 3 -year OS of $82 \%$ for mrEMVI positive cases, which was lesser than the of of $87 \%$ for mrEMVI negative patients, but not statistically significant.

India has a unique profile of rectal cancer presentation, with a high incidence of advanced cancers and aggressive signet ring tumors. In patients with such high-risk features at presentation, the degree of incremental worsening of prognosis due to the presence of mrEMVI is uncertain. We observed a nonsignificant trend to worsened prognosis in mrEMVI positive cases; the lack of significance could be due to the small numbers. EMVI showed significant correlation with DMFS $(p=0.046)$ when the signet ring cell cases were excluded, but again did not correlate with DFS or OS.

A pathological study has demonstrated strong correlation between venous invasion involving thick-walled veins rather than thin-walled veins with respect to the incidence of liver metastases. ${ }^{18}$ Bugg et al and Sohn et $\mathrm{al}^{8,9}$ demonstrated that involvement of larger caliber vessels ( $\geq 3 \mathrm{~mm}$ ) by EMVI leads to increased risk of metastasis. We similarly hypothesized that increased thickness and length of involvement of perirectal vessels by EMVI could correlate with a higher risk of metastases (-Fig. 8). We did not observe a significant difference in the median length and thickness of EMVI of patients who developed distant recurrence and those who did not, although the median length and thickness were slightly higher in the former group. Our numbers were small, and larger studies would be needed to evaluate this finding further.

Our study had a few limitations. It was a retrospective study, although the radiologists were blinded to the histopathology and final outcome. The sample size was small with a limited number of EMVI positive cases. There may also be a referral bias as ours is a tertiary care cancer. Our institute protocol does not include contrast-enhanced study for rectal

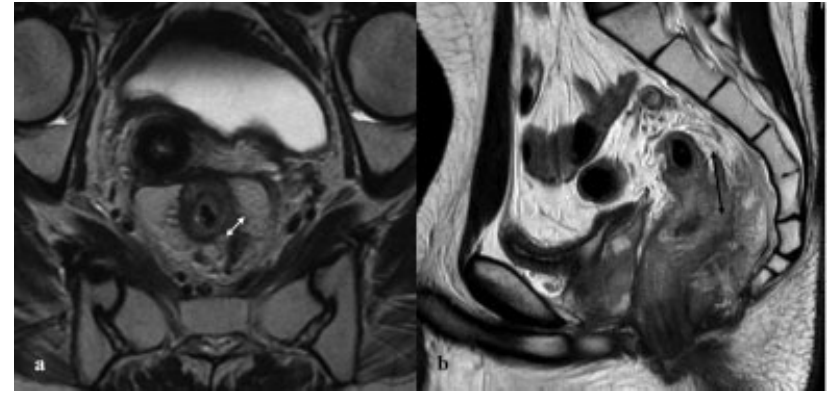

Fig. 8 (a) Axial T2-weighted image showing grade 4 EMVI (white double arrow), measuring $9 \mathrm{~mm}$ in thickness. (b) Sagittal T2-weighted image showing grade 4 EMVI (black double arrow), measuring $36 \mathrm{~mm}$ in length. EMVI, extramural venous invasion.

cancer cases. This is as per the Society of Abdominal Radiology (SAR) and the European Society of Gastrointestinal and Abdominal Radiology (ESGAR) guidelines. However, some studies do suggest better detection of EMVI using gadolinium contrast enhanced sequences. ${ }^{30-33}$ Jhaveri et al found that contrast-enhanced sequences helped in re-stratification of the equivocal Grade 2 EMVI cases which did not show overt tumor signal within a dilated vein. EMVI was seen as a partial or complete filling defect within the contrast-filled vessel lumen, which could then be re-classified as Grade 3 if present or Grade 1 if absent. ${ }^{11,34}$ The ESGAR panel opinion was that dynamic contrast enhanced sequence should be considered a research tool and not adopted in routine practice. The panel acknowledged that it may help in select cases to improve tumor conspicuity in the post-treatment setting and for the assessment of mucinous tumors. ${ }^{30-35}$

In conclusion, we did not find a significant correlation between mrEMVI and DFS or OS in patients with nonmetastatic disease at baseline, although a higher percentage of patients with EMVI developed distant recurrence compared with those without EMVI. Larger multicentric studies are needed to evaluate the significance of these findings. There was, however, a statistically significant worsening of DMFS in non-signet-ring cell cancers.

\section{Financial Support and Sponsorship} None.

\section{Conflict of Interest}

None declared.

\section{References}

1 Consensus Document for Management of Colorectal Cancer ICMR, New Delhi; 2014:11-13

2 Patil PS, Saklani A, Gambhire P, et al. Colorectal cancer in India: an audit from a tertiary center in a low prevalence area. Indian J Surg Oncol 2017;8(04):484-490

3 Nitsche U, Zimmermann A, Späth C, et al. Mucinous and signetring cell colorectal cancers differ from classical adenocarcinomas in tumor biology and prognosis. Ann Surg 2013;258(05):775-782, discussion 782-783

4 Chew MH, Yeo SA, Ng ZP, et al. Critical analysis of mucin and signet ring cell as prognostic factors in an Asian population of 2,764 
sporadic colorectal cancers. Int J Colorectal Dis 2010;25(10): 1221-1229

5 Tamhankar AS, Ingle P, Engineer R, Bal M, Ostwal V, Saklani A. Signet ring colorectal carcinoma: do we need to improve the treatment algorithm? World J Gastrointest Oncol 2016;8(12): 819-825

6 Siddiqui MRS, Simillis C, Hunter C, et al. A meta-analysis comparing the risk of metastases in patients with rectal cancer and MRI-detected extramural vascular invasion (mrEMVI) vs mrEMVI-negative cases. Br J Cancer 2017;116(12):1513-1519

7 Bhangu A, Fitzgerald JE, Slesser A, Northover JM, Faiz O, Tekkis P. Prognostic significance of extramural vascular invasion in $\mathrm{T} 4$ rectal cancer. Colorectal Dis 2013;15(11):e665-e671

8 Bugg WG, Andreou AK, Biswas D, Toms AP, Williams SM. The prognostic significance of MRI-detected extramural venous invasion in rectal carcinoma. Clin Radiol 2014;69(06):619-623

9 Sohn B, Lim JS, Kim H, et al. MRI-detected extramural vascular invasion is an independent prognostic factor for synchronous metastasis in patients with rectal cancer. Eur Radiol 2015;25(05): 1347-1355

10 Smith NJ, Shihab O, Arnaout A, Swift RI, Brown G. MRI for detection of extramural vascular invasion in rectal cancer. AJR Am J Roentgenol 2008;191(05):1517-1522

11 Jhaveri KS, Hosseini-Nik H, Thipphavong S, et al. MRI detection of extramural venous invasion in rectal cancer: correlation with histopathology using elastin stain. AJR Am J Roentgenol 2016;206 (04):747-755

12 Griffin MR, Bergstralh EJ, Coffey RJ, Beart RW Jr, Melton LJ III. Predictors of survival after curative resection of carcinoma of the colon and rectum. Cancer 1987;60(09):2318-2324

13 Compton CC, Greene FL. The staging of colorectal cancer: 2004 and beyond. CA Cancer J Clin 2004;54(06):295-308

14 Balch GC, De Meo A, Guillem JG. Modern management of rectal cancer: a 2006 update. World J Gastroenterol 2006;12(20): 3186-3195

15 Taylor FG, Swift RI, Blomqvist L, Brown G. A systematic approach to the interpretation of preoperative staging MRI for rectal cancer. AJR Am J Roentgenol 2008;191(06):1827-1835

16 Talbot IC, Ritchie S, Leighton MH, Hughes AO, Bussey HJ, Morson $\mathrm{BC}$. The clinical significance of invasion of veins by rectal cancer. Br J Surg 1980;67(06):439-442

17 Shirouzu K, Isomoto $H$, Kakegawa T, Morimatsu M. A prospective clinicopathologic study of venous invasion in colorectal cancer. Am J Surg 1991;162(03):216-222

18 Horn A, Dahl O, Morild I. Venous and neural invasion as predictors of recurrence in rectal adenocarcinoma. Dis Colon Rectum 1991; 34(09):798-804

19 Chand M, Siddiqui MR, Swift I, Brown G. Systematic review of prognostic importance of extramural venous invasion in rectal cancer. World J Gastroenterol 2016;22(04):1721-1726

20 Bokey EL, Chapuis PH, Dent OF, et al. Factors affecting survival after excision of the rectum for cancer: a multivariate analysis. Dis Colon Rectum 1997;40(01):3-10

21 Harrison JC, Dean PJ, el-Zeky F, Vander Zwaag R. From Dukes through Jass: pathological prognostic indicators in rectal cancer. Hum Pathol 1994;25(05):498-505
22 Kim $\mathrm{TH}$, Woo S, Han S, Suh $\mathrm{CH}$, Vargas HA. The diagnostic performance of MRI for detection of extramural venous invasion in colorectal cancer: a systematic review and metaanalysis of the literature. AJR Am J Roentgenol 2019;213(03): 575-585

23 Hunter CJ, Garant A, Vuong T, et al. Adverse features on rectal MRI identify a high-risk group that may benefit from more intensive preoperative staging and treatment. Ann Surg Oncol 2012;19 (04):1199-1205

24 Zhang XY, Wang S, Li XT, et al. MRI of extramural venous invasion in locally advanced rectal cancer: relationship to tumor recurrence and overall survival. Radiology 2018;289(03): 677-685

25 Smith NJ, Barbachano Y, Norman AR, Swift RI, Abulafi AM, Brown G. Prognostic significance of magnetic resonance imagingdetected extramural vascular invasion in rectal cancer. Br J Surg 2008;95(02):229-236

26 Seehaus A, Vaccaro C, Quadrelli M, et al. Magnetic resonance and extramural vascular invasion in patients with rectal cancer and liver metastases. Acta Gastroenterol Latinoam 2015;45(01): 31-36

27 van den Broek JJ, van der Wolf FSW, Heijnen LA, Schreurs WH. The prognostic importance of MRI detected extramural vascular invasion (mrEMVI) in locally advanced rectal cancer. Int J Colorectal Dis 2020;35(10):1849-1854

$28 \mathrm{Gu}$ C, Yang X, Zhang X, et al. The prognostic significance of MRIdetected extramural venous invasion, mesorectal extension, and lymph node status in clinical T3 mid-low rectal cancer. Sci Rep 2019;9(01):12523

29 Sun Y, Li J, Shen L, Wang X, Tong T, Gu Y. Predictive value of MRIdetected extramural vascular invasion in stage $\mathrm{T} 3$ rectal cancer patients before neoadjuvant chemoradiation. Diagn Interv Radiol 2018;24(03):128-134

30 Intven M, Reerink O, Philippens ME. Dynamic contrast enhanced MR imaging for rectal cancer response assessment after neoadjuvant chemoradiation. J Magn Reson Imaging 2015;41(06): 1646-1653

31 Kim SH, Lee JM, Gupta SN, Han JK, Choi BI. Dynamic contrastenhanced MRI to evaluate the therapeutic response to neoadjuvant chemoradiation therapy in locally advanced rectal cancer. J Magn Reson Imaging 2014;40(03):730-737

32 Alberda WJ, Dassen HP, Dwarkasing RS, et al. Prediction of tumor stage and lymph node involvement with dynamic contrast-enhanced MRI after chemoradiotherapy for locally advanced rectal cancer. Int J Colorectal Dis 2013;28(04):573-580

33 Jhaveri KS, Hosseini-Nik H. MRI of rectal cancer: an overview and update on recent advances. AJR Am J Roentgenol 2015;205(01): W42-55

34 Ale Ali H, Kirsch R, Razaz S, et al. Extramural venous invasion in rectal cancer: overview of imaging, histopathology, and clinical implications. Abdom Radiol (NY) 2019;44(01):1-10

35 Beets-Tan RGH, Lambregts DMJ, Maas M, et al. Magnetic resonance imaging for clinical management of rectal cancer: Updated recommendations from the 2016 European Society of Gastrointestinal and Abdominal Radiology (ESGAR) consensus meeting. Eur Radiol 2018;28(04):1465-1475 